\title{
Long-term trends in BMI: are contemporary childhood BMI growth references appropriate when looking at historical datasets?
}

\author{
Richard J Silverwood* \\ David A Leon \\ Bianca L De Stavola \\ Department of Epidemiology and Population Health, London School of \\ Hygiene and Tropical Medicine, Keppel Street, London, WC1E 7HT
}

*Corresponding author. Email: Richard.Silverwood@Ishtm.ac.uk +44 (0)207 9272525

\section{Abstract}

\section{Background}

Body mass index (BMI) is the most widely used surrogate measure of adiposity, and BMI z-scores are often calculated when comparing childhood BMI between populations and population sub-groups. Several growth references are currently used as the basis for calculation of such z-scores, for both contemporary cohorts as well as cohorts born decades ago. Due to the widely acknowledged increases in childhood obesity over recent years it is generally assumed that older birth cohorts would have lower BMls relative to the current standards. However, this reasonable assumption has not been formally tested.

\section{Methods}

Two growth references (1990 UK and $2000 \mathrm{CDC}$ ) are used to calculate BMI z-scores in three historical British national birth cohorts (National Survey of Health and Development (1946), National Child Development Study (1958) and British Cohort Study (1970)). BMI z-scores are obtained for each child at each follow-up age using the LMS method, and their distributions examined.

\section{Results}

Across all three cohorts, median BMI z-score at each follow-up age is observed to be positive in early childhood. This is contrary to what might have been expected given the assumed temporal increase in childhood BMI. However, z-scores then decrease and become negative during adolescence, before increasing once more.

\section{Conclusions}

The differences in BMI distribution between the historical cohorts and the contemporary growth references appear systematic and similar across the cohorts. This might be explained by contemporary reference data describing a faster tempo of weight increase relative to height than observed in older birth cohorts. Comparisons using externally defined $z$-scores over extended periods of time should therefore be interpreted with caution. 


\section{Keywords}

Childhood obesity, body mass index, z-scores, growth references, cohort studies

\section{Introduction}

Body mass index (BMI), calculated as an individual's mass (in kilograms) divided by their height (in metres) squared, has become the most widely used surrogate measure of adiposity. Although BMI has shortcomings, not least the inability to differentiate between lean mass and fat mass (Wells, 2001), it is widely used in pediatrics owing to the ease with which measurements can be made on infants and children, and the often routine manner in which serial anthropometric measurements are recorded.

The use of BMI to investigate adiposity in children is complicated further by the manner in which BMI shows profound changes from birth through to early adulthood (Prentice, 1998), with relationships between the fat and fat-free components of the body being affected by varying growth rates and maturity levels (Maynard et al, 2001).

Z-scores (or standard deviation scores) express measurements in terms of standard deviations from the centre of a distribution and are often used with anthropometric measures, facilitating comparisons across ages in otherwise age-dependent variables.

There exist contemporary BMI growth references, notably the 1990 reference curves for the UK ('1990 UK') and the 2000 Centers for Disease Control and Prevention (CDC) growth charts for the United States ('2000 CDC'), which are frequently used to standardise BMI values. Standardisation of a measurement using an external reference dataset allows an assessment of the position of the measurement within the reference distribution. However, it is unclear whether these growth references are useful as comparisons to less contemporary data. Specifically, given the widely acknowledged increases in childhood BMI over recent years (World Health Organisation, 2000), it may be expected that, on average, childhood BMI in historical datasets would be lower than in the contemporary growth references, leading to a preponderance of negative BMI z-scores. If standardisation does lead to z-scores which do not follow a standard normal distribution, then there may be implications for interpretation of any analysis using these standardised values. For example, if the distribution of BMI z-scores has a mean of -0.5 then an individual with a z-score of 0 , who would normally be considered as having average BMI, would in fact have above average BMI when compared to his or her peers within the same dataset. In this situation it would be important to emphasise that the z-scores are relative to a given standard rather than relative to other subjects within the same dataset, and this may not always be what is wanted.

The aim of the present analysis is to assess the validity of using contemporary BMI growth references when looking at historical British datasets. This is achieved by the calculation and analysis of BMI z-scores using both the 1990 UK and 2000 CDC growth references for three different British national birth cohorts. These cohorts (National Survey of Health and Development (NSHD), National Child Development Study (NCDS) and British Cohort Study (BCS)) are chosen for their national representativeness, range of years of birth (1946-1970), range of ages for which BMI data are available (4-16 years) and longitudinal nature, meaning that the same children can be examined at several follow-up ages in each cohort.

As all three cohorts are made up of children resident in the UK, the 1990 UK growth references would be the more appropriate choice for standardisation of the data. However, as the 2000 CDC growth references would also often be used, their application remains of great interest. 


\section{Materials and methods}

\section{Study samples}

The National Survey of Health and Development (NSHD) is one of the longest running large-scale studies of human development in the world (Medical Research Council NSHD team, 2008). It began as an investigation into the social and economic aspects of 13,687 births occurring during the first week of March 1946 in England, Scotland and Wales. From this original population, a sample consisting of all children whose fathers were non-manual or agricultural workers and a randomly selected one in four sample of children of other manual workers (5362 subjects in total) have been followed up at regular intervals since birth, with children being measured and weighed in underclothes as part of their medical examination (Peckham et al, 1983). A more detailed cohort profile can be found elsewhere (Wadsworth et al, 2006). The present analysis includes follow-up at ages 4, 6, 7, 11 and 15 years. Electronic data for the NSHD were obtained directly from the Medical Research Council NSHD team at University College London.

The National Child Development Study (NCDS) takes as its subjects all the people born in England, Scotland and Wales in one week in March 1958. It has its origins in the Perinatal Mortality Survey, which initially included 17,416 babies (Centre for Longitudinal Studies, 2008a). The cohort was followed up at several ages throughout childhood, with children being measured and weighed in underclothes as part of their medical examination (Peckham et al, 1983). A more detailed cohort profile can be found elsewhere (Power and Elliott, 2006). The present analysis includes follow-up at ages 7, 11 and 16 years. Electronic data for the NCDS were obtained from the UK Data Archive (2007a) and relevant variables identified with the help of the data dictionary provided by the Centre for Longitudinal Studies (2005a).

The British Cohort Study (BCS) takes as its subjects all those living in England, Scotland and Wales who were born in one week in April 1970. Data were collected about the births and families of 16,571 babies, and since then there have been several attempts to gather information from the whole cohort (Centre for Longitudinal Studies, 2008b). Additional people born in the same week, who immigrated to the UK or were identified subsequently, have been added to the cohort. Height and weight at follow-up were measured by school medical staff with a standardised technique (Viner and Cole, 2005). A more detailed cohort profile can be found elsewhere (Elliott and Shepherd, 2006). The present analysis includes measurements at ages 10 and 16 years. Weight was not measured at age 5 years so this follow-up age cannot be included. Electronic data for the BCS were obtained from the UK Data Archive (2007b) and relevant variables identified with the help of the data dictionary provided by the Centre for Longitudinal Studies (2005b).

\section{BMI growth references}

BMI reference curves for UK children were developed for the first time in the mid-1990s (Cole, Freeman and Preece, 1995) based on data collected between 1978 and 1990. Data from 11 distinct surveys were combined, between them recording BMl from birth to age 23, with most being representative of England, Scotland and Wales and all but one being cross-sectional. Summary centile curves were fitted using the LMS method and penalised likelihood (Cole and Green, 1992).

The 2000 CDC growth charts for the United States represent a revised version of the 1977 National Center for Health Statistics (NCHS) growth charts and include BMI-for-age charts (CDC, 2007a). Most of the data came from the National Health and Nutrition Examination Survey (NHANES) cross-sectional studies conducted from 1963 to 1994, though some supplementary data sources were also utilised. Initial curve smoothing for selected major percentiles was accomplished with various parametric and nonparametric procedures, then a normalisation procedure was used to generate z-scores that closely match the smoothed percentile curves (Kuczmarski et al, 2000). 


\section{Statistical analysis}

For every child at each follow-up age in all three cohorts BMI z-scores are calculated using both the 1990 UK and 2000 CDC growth references. For a BMI z-score to be calculated for a given child, and thus for the child to be included in the analysis, data for age, sex and BMI are required. Although each follow-up in each cohort was planned at a specific age, the actual measurements occur over a range of ages. Whilst some variation in age at measurement is unavoidable, it is likely that those at the extremes of the age distribution form a distinct subgroup. For example, subjects measured more than a year after the median age may have been hospitalised for a long period, so their inclusion would undermine the homogeneity of the sample. Thus a further stipulation imposed for these analyses is that all children included at a given follow-up age must have had their measurement within 6 months of the median age at measurement within that follow-up age group. This additionally ensures that when we discuss subjects at a given follow-up age we are referring only to measurements over a 1 year period rather than over a 2-3 year period, which could cause overlap between the follow-up ages.

The calculation of BMI z-scores using the $1990 \mathrm{UK}$ and $2000 \mathrm{CDC}$ growth references uses the LMS method (Cole and Green, 1992). The LMS method summarises the changes in BMI distribution through childhood in a reference dataset by three curves representing the median (M), coefficient of variation (S) and a measure of skewness (L) based on the BoxCox power required to transform the data to normality. The three parameters are constrained to change smoothly with age, and are estimated using penalised maximum likelihood. Once the L, M and S parameters are defined for a reference dataset they can then be used to calculate the BMI value corresponding to any given percentile or z-score, enabling the construction of growth charts. Conversely, given a BMI measurement, the L, M and $\mathrm{S}$ parameters can be used to calculate where, in terms of percentile or z-score, said measurement would occur relative to the distribution of the reference dataset.

The 1990 UK BMI-for-age LMS parameters are extracted from the Microsoft Excel add-in ImsGrowth (Cole and Pan, 2005), with equivalent parameters for the $2000 \mathrm{CDC}$ growth reference obtained via the CDC website (CDC, 2007b).

The $z$-score $(z)$ for a given $B M I$ measurement $(X)$ is calculated as

$$
\mathrm{z}=\frac{(\mathrm{X} / \mathrm{M})^{\mathrm{L}}-1}{\mathrm{LS}} \text { if } \mathrm{L} \neq 0
$$

or

$$
' z=\frac{\log (X / M)}{S} \text { if } L=0,
$$

where $\mathrm{L}, \mathrm{M}$ and $\mathrm{S}$ are the growth reference LMS parameters corresponding to the age and sex of the child.

If the BMI values for a study population agree closely with the growth reference, then the z-scores calculated should be normally distributed with mean 0 and standard deviation 1 . Once calculated, BMI z-scores in each cohort may then be assessed at each follow-up age for any systematic deviation from this (i.e. any systematic difference from the growth reference).

BMI measurements are deemed implausible if they correspond to an absolute z-score (using the 1990 UK growth reference) greater than six and are thus excluded, as has been practiced elsewhere (Tate, Dezateux and Cole, 2006).

We additionally explore whether the observed BMI z-scores in each cohort could be explained by a faster developmental tempo in the (generally) more contemporary BMI references. To do this we construct an artificial dataset by taking the 1990 UK growth reference median BMI values and stretching the timescale using a multiplicative shift of 1.2. We introduce an additional component allowing this slowing down of the developmental tempo to fade away over time, so that by age 18 years the timescale would be back to its original value. As this manipulation results in the lowest point of the BMI curve (the 'BMI rebound') occurring later relative to 1990 UK median, this artificial dataset is referred to as 
the 'delayed BMI rebound cohort'. BMI z-scores for the delayed BMI rebound cohort are calculated using the 1990 UK reference data and the method described above and compared to the BMI z-scores observed in the birth cohorts.

\section{Results}

Table 1 shows the numbers and percentages of subjects included in and excluded from the analysis at each follow-up age. The 'target sample' in each instance is the maximum possible number of individuals for whom data could potentially be collected after the exclusion of the dead, those living abroad and permanent refusals. 'Achieved sample' is the number of individuals for whom at least one response was recorded. 'Sex, age or BMI missing' for an individual means that their BMI z-score cannot be calculated, so they are excluded from the analysis. 'Age > 6 months from follow-up median' for an individual means that the age at which their BMI was observed is not sufficiently similar to the other ages within the age group to allow their inclusion in the analysis.

In the NSHD the achieved sample at each follow-up age was between 89 and $96 \%$ of the target sample. The NCDS includes similarly high levels of achieved sample at each followup age, though a greater degree of missing sex, age or BMI data, particularly at age 16 years $(25 \%)$. Whilst the proportion of the target sample achieved in the BCS was of a similar magnitude to the other cohorts at follow-up age 10 years, at age 16 years the data collection was noticeably reduced by a teachers' strike (Elliott and Shepherd, 2006).

Additionally, over $50 \%$ of children have either sex, age or BMI values missing so cannot be included in the analysis, meaning that at age 16 years the BCS cannot be considered as nationally representative as the other cohorts.

From Table 1 it can be seen that the requirement for data to have been recorded within 6 months of the median age at each follow-up age rarely results in the exclusion of a significant amount of data and never more than $4 \%$ of the achieved sample.

The percentage of males and summaries of the age and BMI distributions for the subjects who are included in the analysis are shown in Table 2. In each cohort at each follow-up age, except the less-representative age 16 years follow-up in the BCS, there are slightly more males than females. Due to the skewed nature of the age and BMI distributions, medians and inter-quartile ranges (IORs) are presented. Both the magnitude and the variability of BMI can be seen to increase after about age 7 years.

It is possible to calculate the years of birth of the children included in the reference datasets by examining the data collection dates and subject ages (Cole, Freeman and Preece, 1995; Kuczmarski et al, 2000). These are presented in Table 3 for each age considered. Across all the ages, UK 1990 reference data were collected from subjects born 1962-84 and CDC 2000 reference data were collected from subjects born 1950-90. Thus, whilst all the data for both $\mathrm{BMI}$ references were collected from subjects born after those in the NSHD (in many case quite considerably after), for the other birth cohorts this is not always the case. At ages 11 and 16 years some of the subjects in the CDC 2000 reference dataset were born a little earlier than the NCDS subjects. Nearly all the subjects in the UK 1990 reference dataset were born after the BCS subjects at age 10 years, but almost all the CDC 2000 children were born before. At age 16 years all or virtually all the subjects in both BMI references were born before the BCS subjects. 


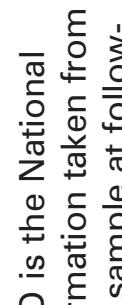

오은 뉸

公吉

बं वें

을 党

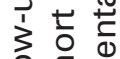

은

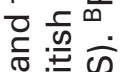

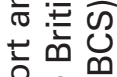

응 壬

도융

는

ลำ 잉

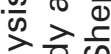

宁 离

Ф) 듬

등 응 음

능 응 둥

ర్ల \

음

खx

. $\frac{1}{0}$

융 응

는

我产

엉.믕 잉

윽 $\frac{5}{5} \frac{0}{0}$

क人民ँ

क 世

Ð

인응 원

ఎ ญ

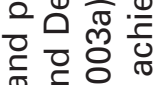

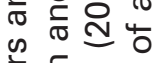

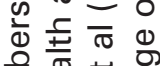

ह 웡

之先至过

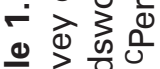

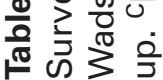

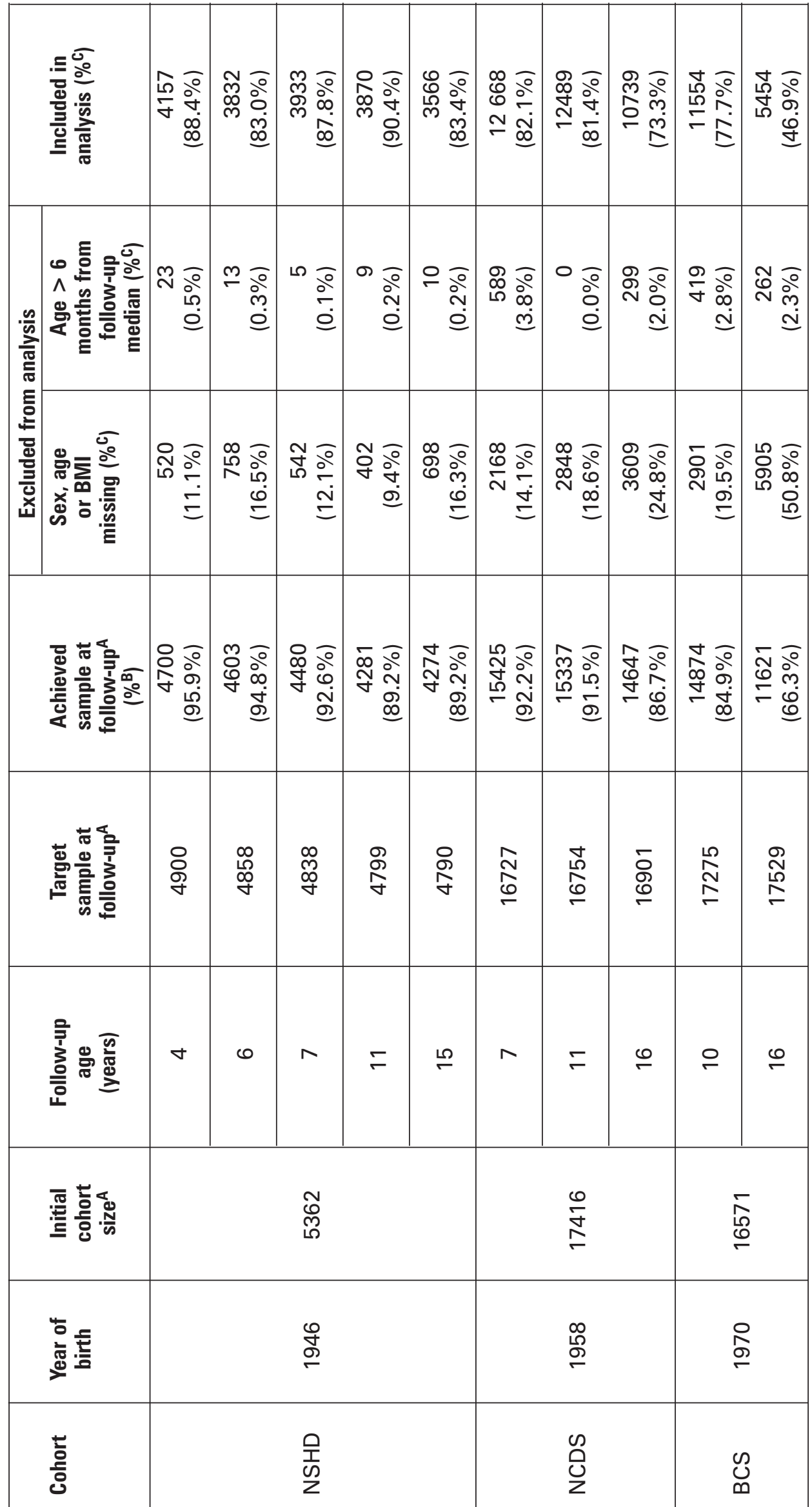




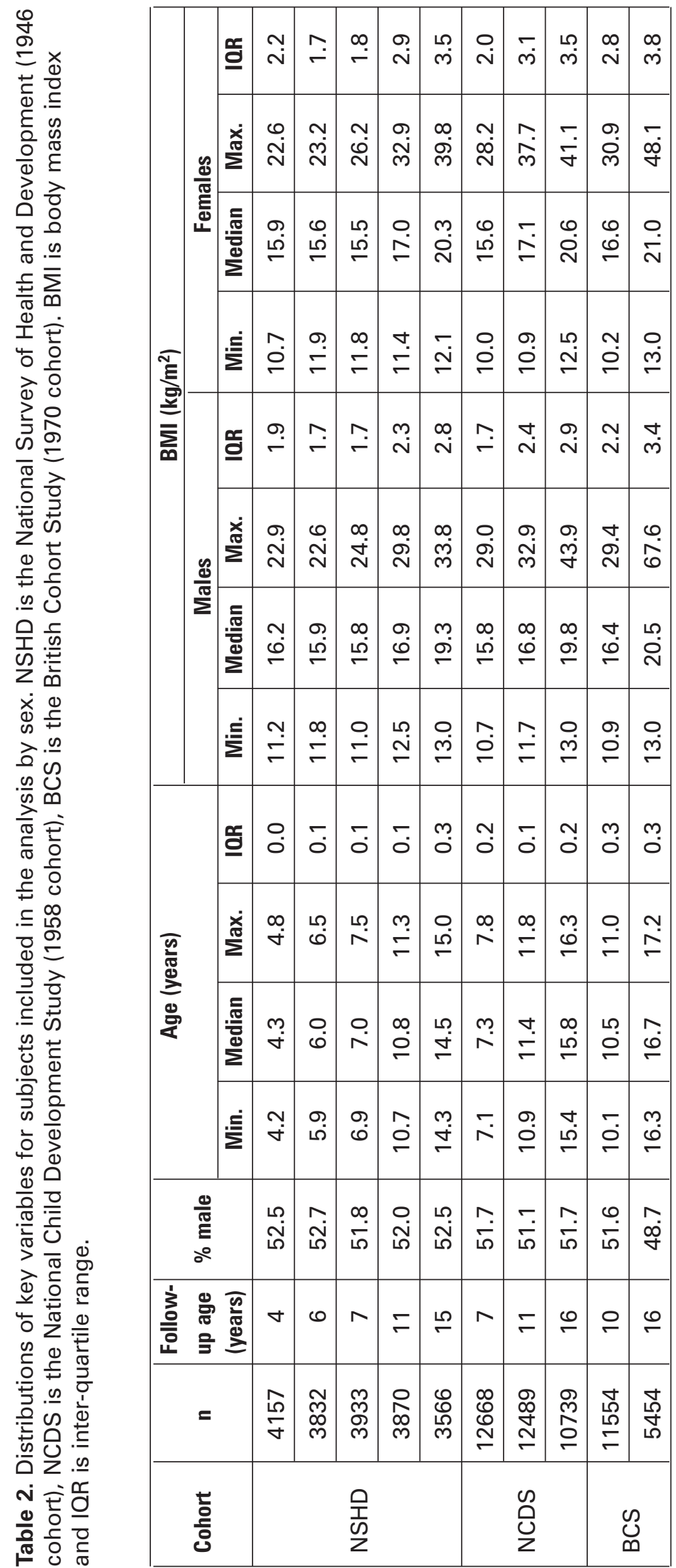


The distributions of the calculated BMI z-scores for each birth cohort using the 1990 UK and 2000 CDC growth references are shown in Table 4 and Table 5, respectively. Once more, medians and IQRs are presented due to the skewed nature of the distributions. There is clearly a great deal of variation in the median values of BMI z-score in the cohorts at different follow-up ages. Median z-scores are generally positive in early childhood before decreasing, often becoming negative, then increasing once more. These results are more easily interpretable when plotted graphically.

Table 3. Ranges of years of birth of subjects in each reference dataset for each age considered in the present analysis.

\begin{tabular}{|l|c|c|}
\hline BMI reference & Age (years) & Range of years of birth \\
\hline \multirow{4}{*}{ UK 1990 } & 4 & 1983 \\
\cline { 2 - 3 } & 6 & $1972-84$ \\
\cline { 2 - 3 } & 7 & $1971-83$ \\
\cline { 2 - 3 } & 10 & $1968-80$ \\
\cline { 2 - 3 } & 11 & $1967-79$ \\
\cline { 2 - 3 } & 15 & $1963-71$ \\
\hline \multirow{4}{*}{ CDC 2000 } & 16 & $1962-71$ \\
\cline { 2 - 3 } & 4 & $1967-90$ \\
\cline { 2 - 3 } & 6 & $1957-88$ \\
\cline { 2 - 3 } & 7 & $1956-73$ \\
\cline { 2 - 3 } & 10 & $1953-70$ \\
\cline { 2 - 3 } & 11 & $1952-69$ \\
\cline { 2 - 3 } & 15 & $1951-65$ \\
\hline
\end{tabular}

Table 4. Distributions of calculated body mass index (BMI) z-scores using the 1990 United Kingdom (UK) growth reference, by sex. NSHD is the National Survey of Health and Development (1946 cohort), NCDS is the National Child Development Study (1958 cohort) and BCS is the British Cohort Study (1970 cohort). Results weighted to adjust for the one in four sampling of children from manual and self-employed workers.

\begin{tabular}{|c|c|c|c|c|c|c|c|c|c|}
\hline \multirow{3}{*}{ Cohort } & \multirow{3}{*}{$\begin{array}{l}\text { Follow- } \\
\text { up age } \\
\text { (years) }\end{array}$} & \multicolumn{8}{|c|}{ BMI z-score } \\
\hline & & \multicolumn{4}{|c|}{ Males } & \multicolumn{4}{|c|}{ Females } \\
\hline & & Min. & Median & Max. & IOR & Min. & Median & Max. & IQR \\
\hline \multirow{5}{*}{ NSHD } & 4 & -5.59 & 0.41 & 3.85 & 1.45 & -5.44 & 0.21 & 3.40 & 1.41 \\
\hline & 6 & -4.14 & 0.29 & 3.23 & 1.29 & -3.07 & 0.08 & 3.14 & 1.06 \\
\hline & 7 & -5.33 & 0.16 & 3.44 & 1.17 & -3.13 & -0.07 & 3.32 & 1.10 \\
\hline & 11 & -3.55 & 0.02 & 3.16 & 1.13 & -4.43 & -0.19 & 3.37 & 1.35 \\
\hline & 15 & -4.70 & 0.08 & 3.15 & 1.20 & -5.30 & 0.23 & 3.63 & 1.30 \\
\hline \multirow{3}{*}{ NCDS } & 7 & -5.85 & 0.14 & 4.09 & 1.15 & -5.57 & -0.08 & 3.65 & 1.18 \\
\hline & 11 & -4.88 & -0.14 & 3.37 & 1.29 & -5.28 & -0.28 & 3.73 & 1.45 \\
\hline & 16 & -5.15 & 0.01 & 3.84 & 1.24 & -5.10 & 0.08 & 3.69 & 1.28 \\
\hline \multirow{2}{*}{ BCS } & 10 & -5.75 & -0.16 & 3.17 & 1.19 & -5.97 & -0.27 & 3.18 & 1.35 \\
\hline & 16 & -5.50 & 0.03 & 4.66 & 1.37 & -4.89 & 0.09 & 4.15 & 1.37 \\
\hline
\end{tabular}


Table 5. Distributions of calculated body mass index (BMI) z-scores using the 2000 Centers for Disease Control and Prevention (CDC) growth reference, by sex. NSHD is the National Survey of Health and Development (1946 cohort), NCDS is the National Child Development Study (1958 cohort) and BCS is the British Cohort Study (1970 cohort). Results weighted to adjust for the one in four sampling of children from manual and self-employed workers.

\begin{tabular}{|c|c|c|c|c|c|c|c|c|c|}
\hline \multirow{3}{*}{ Cohort } & \multirow{3}{*}{$\begin{array}{l}\text { Follow- } \\
\text { up age } \\
\text { (years) }\end{array}$} & \multicolumn{8}{|c|}{ BMI z-score } \\
\hline & & \multicolumn{4}{|c|}{ Males } & \multicolumn{4}{|c|}{ Females } \\
\hline & & Min. & Median & Max. & IOR & Min. & Median & Max. & IOR \\
\hline \multirow{5}{*}{ NSHD } & 4 & -6.46 & 0.53 & 3.70 & 1.49 & -8.35 & 0.50 & 2.84 & 1.38 \\
\hline & 6 & -5.07 & 0.36 & 2.50 & 1.23 & -3.89 & 0.25 & 2.46 & 1.05 \\
\hline & 7 & -7.04 & 0.18 & 2.52 & 1.11 & -3.83 & 0.06 & 2.49 & 1.10 \\
\hline & 11 & -3.90 & -0.12 & 2.33 & 1.05 & -4.68 & -0.17 & 2.48 & 1.22 \\
\hline & 15 & -5.03 & -0.13 & 2.37 & 1.10 & -5.54 & 0.21 & 2.49 & 1.08 \\
\hline \multirow{3}{*}{ NCDS } & 7 & -7.90 & 0.15 & 2.79 & 1.10 & -8.05 & 0.04 & 2.64 & 1.17 \\
\hline & 11 & -5.42 & -0.29 & 2.46 & 1.20 & -5.61 & -0.24 & 2.66 & 1.30 \\
\hline & 16 & -5.51 & -0.21 & 2.87 & 1.15 & -5.47 & 0.08 & 2.47 & 1.08 \\
\hline \multirow{2}{*}{$\mathrm{BCS}$} & 10 & -6.75 & -0.28 & 2.33 & 1.13 & -6.73 & -0.24 & 2.38 & 1.24 \\
\hline & 16 & -5.98 & -0.23 & 3.43 & 1.27 & -5.45 & 0.06 & 2.59 & 1.16 \\
\hline
\end{tabular}

Fig. 1 and Fig. 2 show the median BMI z-score plotted against the median age at each follow-up age in the three cohorts. Fig. 1 displays the BMI z-scores calculated using the 1990 UK (upper plot) and 2000 CDC (lower plot) growth references for males, and Fig. 2 shows the equivalent plots for females. Whilst the four plots show all three cohorts to exhibit broadly similar patterns of BMI z-score throughout childhood, there are some cohort-, sex- and growth reference-specific features.

For the males of all three cohorts, using the 1990 UK growth reference (Fig. 1, upper plot) results in a median BMI z-score that is positive but decreasing through early childhood, reaching a minimum around age 11 years before increasing once more. In the NSHD (the earliest birth cohort) this minimum value corresponds to a BMI z-score of approximately zero, whereas in the other cohorts the minima are clearly negative. Use of the 2000 CDC growth references (Fig. 1, lower plot) results in a similar pattern of median BMI z-score through early childhood. In this case, however, all three cohorts cross into negativity, with more extreme minimum values exhibited, then, rather than returning to positivity, they merely level off and remain negative.

Over the age range for which data are available for more than one cohort a cross-cohort comparison can be made. It can be seen that at age 6-7 years the median BMI z-scores for the NSHD and NCDS are very similar whereas at later ages it is the NCDS and the BCS that take similar values with those for the NSHD clearly greater, especially around age 11 years. 

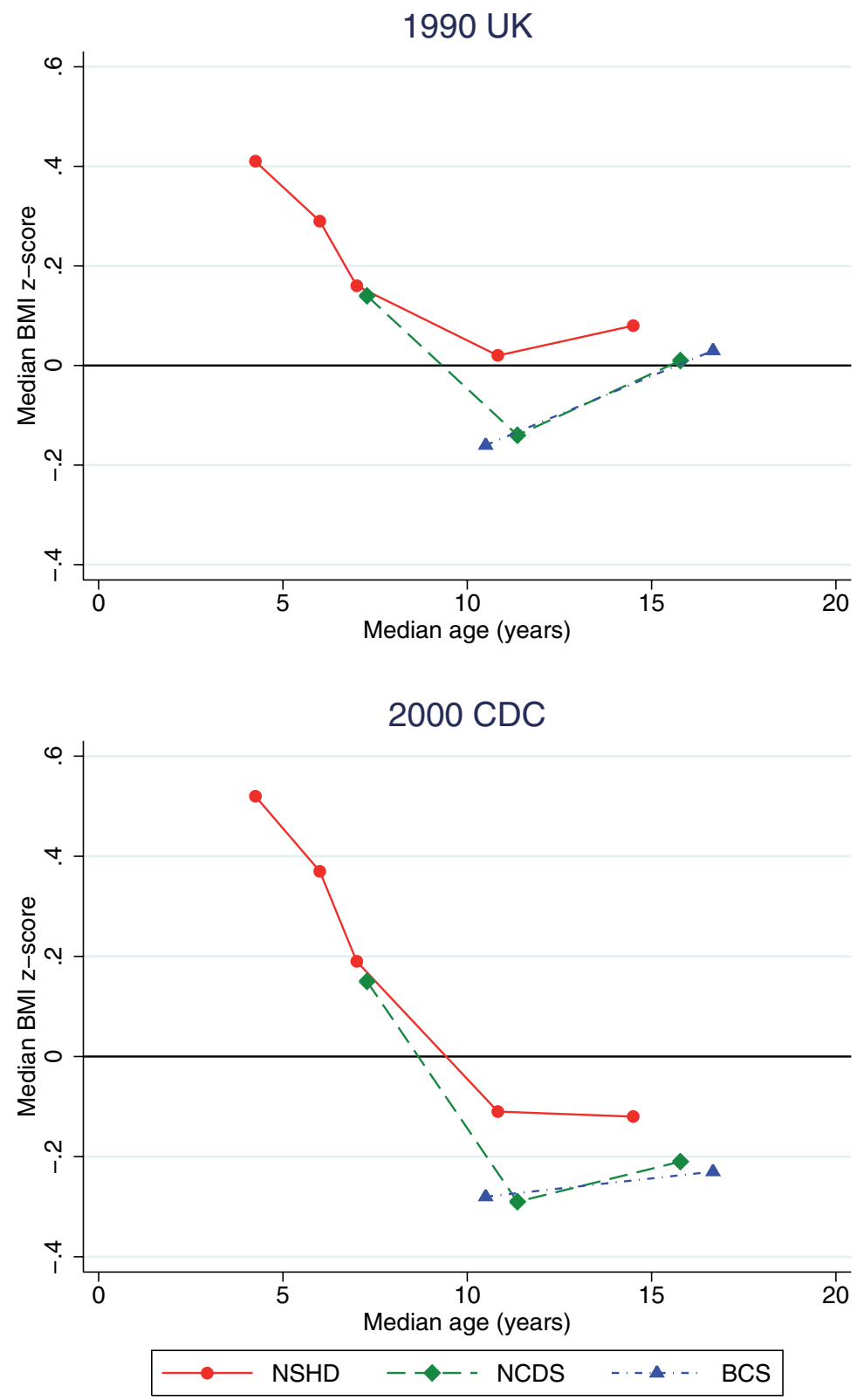

Figure 1. Plots of body mass index (BMI) z-score calculated using the 2000 Centers for Disease Control and Prevention (CDC) (upper plot) and 1990 United Kingdom (UK) (lower plot) growth references against age for males. NSHD is the National Survey of Health and Development (1946 cohort), NCDS is the National Child Development Study (1958 cohort) and BCS is the British Cohort Study (1970 cohort). 

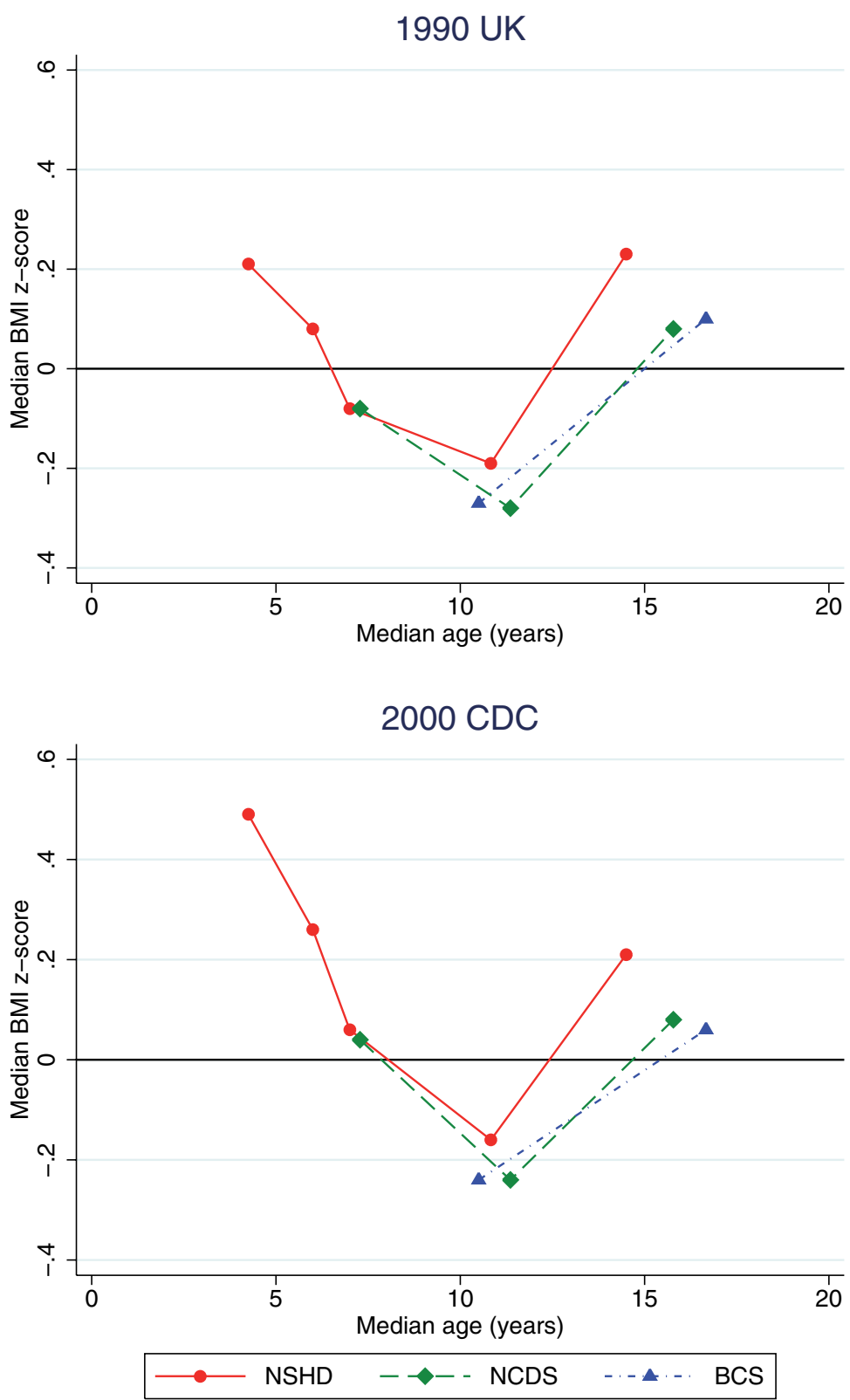

Figure 2. Plots of body mass index (BMI) z-score calculated using the 2000 Centers for Disease Control and Prevention (CDC) reference (upper plot) and 1990 United Kingdom (UK) (lower plot) growth references against age for females. NSHD is the National Survey of Health and Development (1946 cohort), NCDS is the National Child Development Study (1958 cohort) and BCS is the British Cohort Study (1970 cohort). 
Median BMI z-scores at each follow-up age in females are plotted in Fig. 2. Under both growth references the median BMI z-score is positive though decreasing through early childhood, before crossing into negativity, with all three cohorts reaching a minimum of about -0.2 around age 11 years. Median BMI z-scores then increase once more to exhibit positive values in adolescence. The main difference between the two plots in Fig. 2 is that when using the $2000 \mathrm{CDC}$ growth reference the median BMI z-score is noticeably greater through early childhood, resulting in it becoming negative slightly later, though generally the growth reference-specific differences are less marked than in the males.

In terms of the differences between the cohorts within each plot, the pattern is somewhat similar to that seen for the males, with median BMI z-score in the NSHD and the NCDS similar at age 6-7 years, then median BMI z-score in the NSHD becoming increasingly greater than in the other two cohorts at older ages.

Whilst the overall trends in median BMI z-score profile are clearly similar under the two growth references, there are some differences. The 2000 CDC reference data appear to decrease the z-score value relative to the 1990 UK data somewhat in males at follow-up ages of 10 years and older, whereas females of follow-up age 7 years and younger see an increased BMI z-score. These observations correspond to the differences between the growth references evident in Fig. 3, showing median BMI in each growth reference plotted against age, for males (upper plot) and females (lower plot). The upper plot shows that up to approximately age 8 years, the two medians for the males are very similar, but then the 2000 CDC median becomes noticeably and increasingly greater than the 1990 UK median. This means that males of this age would have a reduced BMI z-score if calculated with the 2000 CDC reference data. However, in females (lower plot), it is between the ages of approximately 3 and 10 years that there is a difference between the two reference medians, with the 2000 CDC median being the lower in this instance. This results in any BMI z-scores calculated over this age range being greater when using the $2000 \mathrm{CDC}$ reference data.

In order to explore whether the observed BMI z-scores in each cohort could be explained by a faster developmental tempo in the (generally) more contemporary BMI references, we construct an artificial cohort with a reduced developmental tempo (the 'delayed BMI rebound cohort') as described in the Methods section. The upper plot in Fig. 4 is of BMI against age for the 1990 UK growth reference median and the median of the delayed BMI rebound cohort. It can be seen that the median age at which the BMI rebound occurs has been delayed from 5.9 years in the 1990 UK growth reference to 6.7 years. BMI z-scores for the artificially constructed cohort (calculated using the 1990 UK reference) are presented in the lower part of Fig. 4, plotted against age. The similarity between this plot and many of the equivalent plots for the cohorts included in this analysis is apparent. 

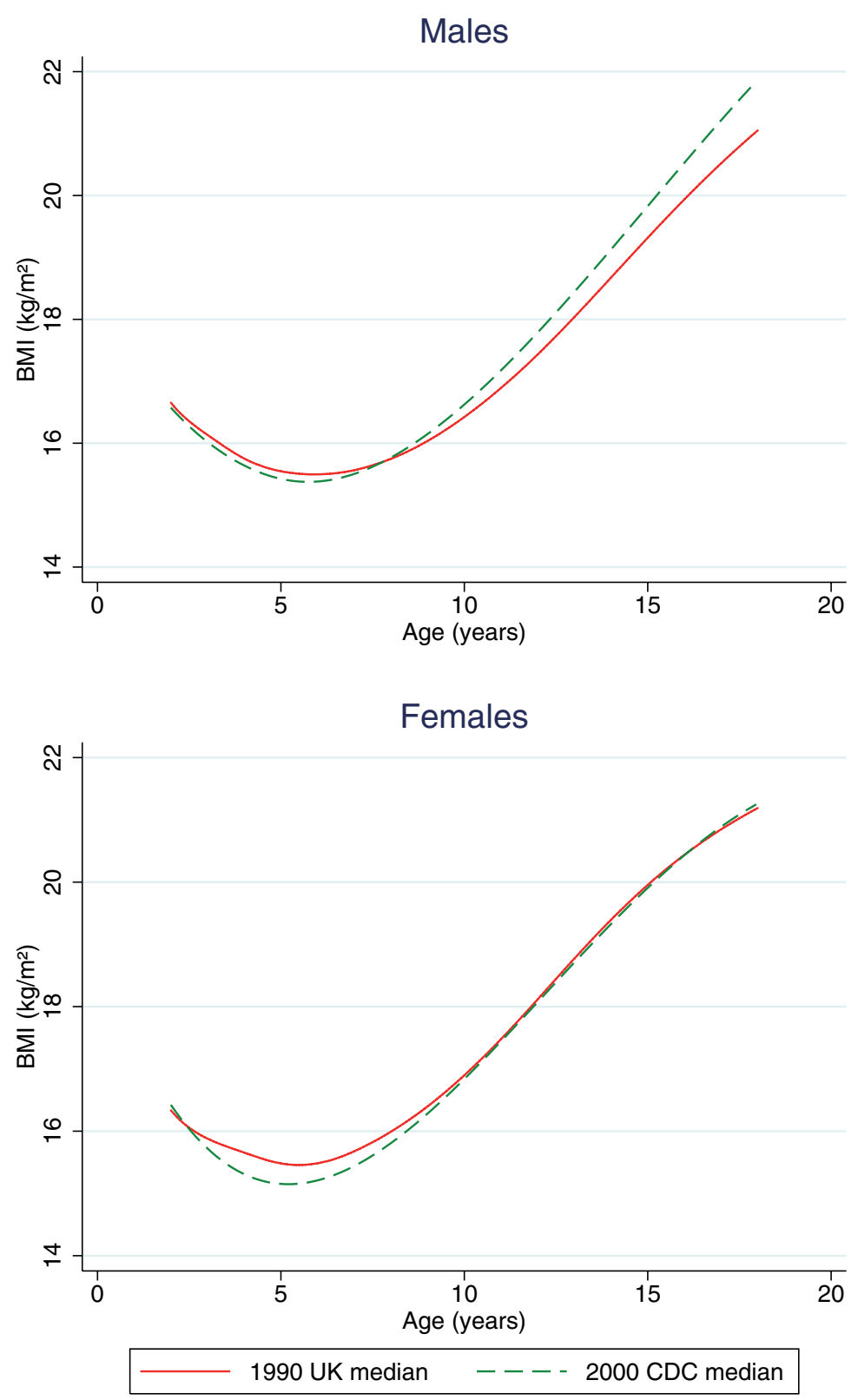

Figure 3. Plots of median body mass index (BMI) in the 1990 United Kingdom (UK) and 2000 Centers for Disease Control and Prevention (CDC) growth references against age for males (upper plot) and females (lower plot). 

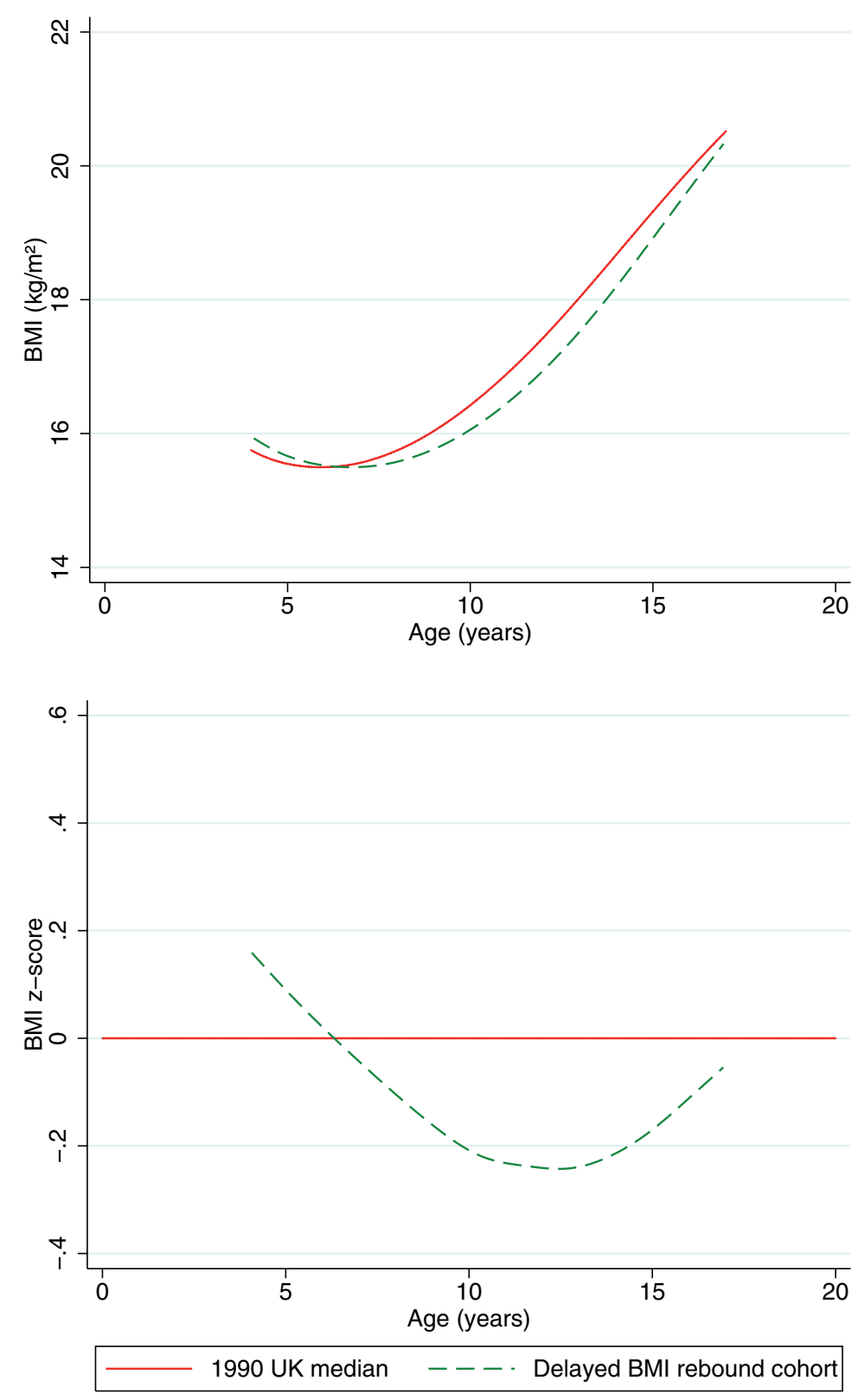

Figure 4. Plots of body mass index (BMI) (upper plot) and body mass index z-score calculated using the 1990 United Kingdom (UK) growth reference (lower plot) against age for the artificially constructed delayed BMI rebound cohort. 


\section{Discussion}

This analysis has uncovered a tendency for historical cohorts of children born between 1946 and 1970 to differ in terms of BMI distribution from both the 1990 UK and 2000 CDC growth references. Moreover, the deviations exhibited are systematic and largely similar between the historical cohorts. All three cohorts have positive but decreasing BMI z-score through early childhood. There is a general trend for z-scores to become negative in the pre-pubertal period, attaining a minimum value in early puberty, before beginning to increase once more, most markedly in females, in the late-pubertal period.

The proportion of subjects included in the analysis at each follow-up age in each cohort is always somewhat less than $100 \%$. Whilst this could lead to the analysed subjects not being nationally representative, the fact that the changes in BMI z-score with increasing age are not in a consistent direction suggests that they are not merely a result of selective missingness. We thus believe that the patterns in BMI z-score observed are genuine.

Given the widely acknowledged obesity 'epidemic' evident over recent years (World Health Organisation, 2000), it may be expected that the calculation of z-scores in historical cohorts using more contemporary reference data would lead to negative values in the majority of subjects. All the NSHD subjects, and those at the younger follow-up ages in the NCDS, were born before both sets of reference subjects in the present analysis, but consistently negative BMI z-scores are not observed. Similarly, as the BCS subjects were born around the same time or later than the reference subjects, one may anticipate their BMI z-scores to consistently lie close to zero or even be positive. However, their BMI z-scores are clearly negative at age 10 years for both sexes and both BMI references, remaining so at age 16 years for males using the $2000 \mathrm{CDC}$ reference.

It may also be expected that the historical cohorts show a temporal ordering, with those born more recently having relatively higher BMI z-scores. In addition to its less temporal proximity, one may additionally expect childhood BMI in the NSHD to be lower than in the other cohorts due to cohort members' nutrition being influenced by food rationing, which continued after the war until 1954 (Hollingsworth, 1961). Although the cross-cohort comparisons are limited by the short range of ages for which data are available for all the cohorts, it is clear that no such temporal ordering exists. Indeed, BMI z-score in the NSHD is almost always higher than that seen in the other cohorts. Previous analyses have found BMI to be similar between NSHD and NCDS at age 7 years (Li et al, 2008) and at age 11 years (Wadsworth et al, 2003b), which is in agreement with our observations. It has also been suggested that BCS subjects have considerably larger BMI at age 10-11 years than subjects in the other two cohorts (Wadsworth et al, 2003b), but we do not find this in the present analysis.

To expect patterns in BMI z-score through childhood in the historical cohorts predating the reference datasets to be merely 'negative' may be something of an over-simplification. For the median BMI z-score in a given cohort to take a constant value of, say, -0.2 across the entire range of ages would mean the median BMI within the cohort being equal to the median BMI in the reference dataset minus 0.2 of a standard deviation at each follow-up age. Implicit in this is that the median BMI growth trajectories in the historical cohort and the reference dataset follow the same shape. However, acknowledged secular changes in growth patterns over the last century, particularly a trend towards a faster developmental tempo (Cole, 2003), mean this may not be true.

One way to describe the BMI growth trajectory is by the timing of the BMI rebound, with a secular increase in developmental tempo being evidenced by an advancing BMI rebound. Indeed, precisely this trend has been found in Swedish children between 1973-5 and 19857 (Eriksson, Rasmussen and Nordqvist, 2005). Through the construction and analysis of a delayed BMI rebound cohort, we have shown that the results observed in the present analysis could plausibly be explained by more rapid development in the reference data relative to that seen in the historical cohorts.

An earlier BMI rebound has been shown to be associated with increased BMI in adolescence and adulthood (Rolland-Cachera et al, 1984; Rolland-Cachera et al, 1987). Although the growth references and historical cohorts examined here are not truly 
comparable in the same way as, say, two individuals in the same cohort, it is conceivable that a similar mechanism could be at work, with the earlier BMI rebound of the reference data leading to increased $\mathrm{BMI}$ at a later date. In this way, the positive z-scores evident in early childhood could be attributed solely to the earlier BMI rebound in the reference data, with the possibility of greater $\mathrm{BMI}$ in adulthood in the more contemporary reference dataset bringing the findings more in line with recent trends. This does, however, conflict somewhat with the positive z-scores around age 15 years, particularly in the NSHD, which remain more difficult to explain.

It is also informative to consider the observed patterns of BMI z-scores in the historical cohorts in terms of the corresponding height and weight z-scores. Calculation of these zscores using an analogous method to that for BMI shows that both are almost always negative, though height $z$-scores are generally more negative than weight z-scores. In particular, the ages at which positive BMI z-scores are observed correspond to periods where weight z-scores are close to zero and height z-scores are more strongly negative, meaning subjects in the historical cohorts have slightly lower weight than those in the references samples, but are much shorter.

The observed trends could certainly affect analyses which use z-scores calculated using the UK 1990 or CDC 2000 BMI references. Take, for example, a female in the NSHD with a BMI of $15.5 \mathrm{~kg} / \mathrm{m}^{2}$ at age 4 years, $17.8 \mathrm{~kg} / \mathrm{m}^{2}$ at age 11 years and $19.1 \mathrm{~kg} / \mathrm{m}^{2}$ at age 15 years. These BMI values correspond respectively to the 38th, 64th and 32nd centiles of the observed BMI values in the NSHD at each follow-up age. Standardised using the CDC 2000 $\mathrm{BMI}$ reference, all three measurements correspond to a $z$-score of +0.2 . This subject would thus be considered as maintaining the same relative BMI throughout childhood. However, when compared to her own peers within the NSHD this is clearly not quite the case. If females following a similar BMI trajectory to this were found to, say, be at increased risk of a certain adverse health outcome, then it is possible that using the z-scores rather than the observed BMI values themselves may lead to a slightly different interpretation.

If BMI z-scores are being used in an analysis as a means of comparing the observed BMI measurements to those in the reference dataset, then external standardisation using that reference dataset is a valid approach. If z-scores are merely required to rescale BMI, however, then standardisation using an external reference dataset may provide misleading results, as seen above. One potential alternative is to use internal standardisation, where the z-score for a subject is calculated based on the position of their measurement relative to those of their peers within the same dataset. This could be achieved using the LMS method (Cole and Green, 1992) to effectively construct a growth chart based on the observed data. For a sufficiently large dataset this approach will provide normally distributed z-scores with mean zero, though it is not recommended for very small datasets. In these instances, external standardisation may remain the only viable option.

To conclude, if children in historical datasets and children in growth references do not have similar distributions of measurements at a given age, then the resultant z-scores will not follow the expected standard normal distribution. When comparisons are to be made across ages, and the differences between the distributions of measurements in the historical and reference datasets are not broadly consistent at each age, for example due to different underlying growth trajectories, then analyses may potentially be misleading. Unless any differences in the age-specific distributions are explored and acknowledged, for example by reporting details of the distribution of the calculated z-scores, externally defined z-scores should be viewed with caution.

\section{Acknowledgements}

We would like to thank Professor Diana Kuh of the MRC Unit for Lifelong Health and Ageing for providing the data from the National Survey of Health and Development. The National Child Development Study and British Cohort Study data were obtained from the UK Data Archive, funded by the Economic and Social Research Council. Further thanks to Debbie Lawlor, Tim Cole and Russell Viner for helpful advice and feedback. 


\section{References}

Centers for Disease Control and Prevention. (2007a) CDC Growth Charts: United States. http://www.cdc.gov/nchs/about/major/nhanes/growthcharts/background.htm.

Centers for Disease Control and Prevention. (2007b) BMI-for-age charts, 2 to 20 years, LMS parameters and selected smoothed BMI (kilograms/meters squared) percentiles, by sex and age. http://www.cdc.gov/nchs/data/nhanes/growthcharts/bmiage.txt.

Centre for Longitudinal Studies. (2005a) National Child Development Study Data Dictionary. http://www.cls.ioe.ac.uk/studies.asp?section $=0001000200030014$

Centre for Longitudinal Studies. (2005b) British Cohort Study Data Dictionary. http://www.cls.ioe.ac.uk/studies.asp?section $=0001000200020012$.

Centre for Longitudinal Studies. (2008a) National Child Development Study. http://www.cls.ioe.ac.uk/studies.asp?section $=000100020003$.

Centre for Longitudinal Studies. (2008b) British Cohort Study. http://www.cls.ioe.ac.uk/studies.asp?section $=000100020002$.

Cole TJ. (2003) The secular trend in human physical growth: a biological view. Economics and Human Biology, 1, 161-8.

Cole TJ, Freeman JV and Preece MA. (1995) Body mass index reference curves for the UK, 1990. Archives of Disease in Childhood, 73, 25-9.

Cole TJ and Green PJ. (1992) Smoothing reference centile curves: the LMS method and penalized likelihood. Statistics in Medicine, 11, 1305-19.

Cole TJ and Pan H. (2005) ImsGrowth version 2.12. Medical Research Council.

Elliott J and Shepherd P. (2006) Cohort profile: 1970 British Birth Cohort (BCS70). International Journal of Epidemiology, 35, 836-43.

Eriksson M, Rasmussen F and Nordqvist T. (2005) Changes in shape and location of BMI distributions of Swedish children. Acta Paediatrica, 94, 1558-65.

Hollingsworth DF. (1961) The changing patterns in British food habits since the 1939-45 war. Proceedings of the Nutrition Society, 20, 25-30.

Kuczmarski RJ, Ogden CL, Grummer-Strawn LM, Flegal KM, Guo SS, Wei R, Mei Z, Curtin LR, Roche AF and Johnson CL. (2000) CDC growth charts: United States. Advance Data, 314.

Li L, Hardy R, Kuh D, Lo Conte R and Power C. (2008) Child-to-adult body mass index and height trajectories: a comparison of 2 British birth cohorts. American Journal of Epidemiology, 168: 1008-15.

Maynard LM, Wisemandle W, Roche AF, Chumlea WC, Guo SS and Siervogel RM. (2001) Childhood body composition in relation to body mass index. Pediatrics, 107, 344-50.

Medical Research Council NSHD team. (2008) University College London. National Survey of Health and Development (NSHD). http://www.nshd.mrc.ac.uk

Peckham CS, Stark O, Simonite V and Wolff OH. (1983) Prevalence of obesity in British children born in 1946 and 1958. British Medical Journal, 286, 1237-42. 
Power C and Elliott J. (2006) Cohort profile: 1958 British birth cohort (National Child Development Study). International Journal of Epidemiology, 35, 34-41.

Prentice AM. (1998) Body mass index standards for children: Are useful for clinicians but not yet for epidemiologists. British Medical Journal, 317, 1401-2.

Rolland-Cachera MF, Deheeger M, Bellisle F, Sempe M, Guilloud-Bataille M and Patois E. (1984) Adiposity rebound in children: a simple indicator for predicting obesity. American Journal of Clinical Nutrition, 39, 129-35.

Rolland-Cachera MF, Deheeger M, Guilloud-Bataille M, Avons P, Patois E and Sempe M. (1987) Tracking the development of adiposity from one month of age to adulthood. Annals of Human Biology, 14, 219-29.

Tate AR, Dezateux C and Cole TJ. (2006) Is infant growth changing? International Journal of Obesity, 30, 1094-6.

UK Data Archive. (2007a) National Child Development Study datasets.

http://www.data-archive.ac.uk/findingData/ncdsTitles.asp.

UK Data Archive. (2007b) 1970 British Cohort Study datasets.

http://www.data-archive.ac.uk/findingData/bcsTitles.asp.

Viner RM and Cole TJ. (2005) Adult socioeconomic, educational, social, and psychological outcomes of childhood obesity: a national birth cohort study. British Medical Journal, 330, 1354.

Wadsworth ME, Butterworth SL, Hardy RJ, Kuh DJ, Richards M, Langenberg C, Hilder WS and Connor M. (2003a) The life course prospective design: an example of benefits and problems associated with study longevity. Social Science \& Medicine, 57, 2193-205.

Wadsworth M, Butterworth S, Montgomery S, Ehlin, A and Bartley, M. (2003b) Health. In: Ferri E, Bynner J, Wadsworth M. eds. Changing Britain, changing lives: three generations at the turn of the century. Pp 207-36. Institute of Education Press, London.

Wadsworth M, Kuh D, Richards M and Hardy R. (2006) Cohort profile: The 1946 National Birth Cohort (MRC National Survey of Health and Development). International Journal of Epidemiology, 35, 49-54.

Wells JCK. (2001) A critique of the expression of paediatric body composition data. Archives of Disease in Childhood. 85, 67-72.

World Health Organisation. (2000) Obesity: preventing and managing the global epidemic. WHO Technical Report Series. 